\title{
Las representaciones sociales de la dependencia
}

\section{Eguzki Urteaga}

Dpto. de Sociología I

Euskal Herriko Unibertsitatea / Universidad del País Vasco

<eguzki.urteaga@ehu.es>

\begin{abstract}
Autonomia indibidualak eta pertsonen arteko berdintasunak arau moralak eta politika nagusiak osatzen dituzten gizarte batean eta non arazo horren inguruan eztabaida publiko bat dagoen, menpekotasunaren irudipen sozialak aztertzea ezinbestekoa da, horrek ondorengoa suposatzen duelarik: 1) menpekotasuna pentsatzea, jakiteko ea modelo propiorik dagoen; 2) menpekotasuna eta ezgaitasunaren arteko lotura jorratzea; 3) menpekotasuna porrotari lotzen duen pertzepzioa lantzea, gizarte politiken eragin anbibalenteetan ekinez; 4) lan hauskortasuna eta menpekotasuna lotzeko joera aztertzea. Artikulu hau menpekotasunaren alderdi anitza eta aldakorra agerian uzten ahalegintzen da, jakinik ez dela ezgaituetara, adineko pertsonetara ezta ere gaur egunera mugatzen.
\end{abstract}

\section{GILTZA HITZAK:}

menpekotasuna, irudipen soziala, feminizazioa, etnizazio, care.
En una sociedad donde la autonomía individual y la igualdad entre personas constituyen normas morales y políticas dominantes y se produce un debate público en torno a esta cuestión, es preciso analizar las representaciones sociales de la dependencia, lo que supone: 1) pensar la dependencia, para determinar si existe un modelo específico; 2) estudiar la asociación entre dependencia e incapacidad; 3) detenerse sobre la percepción de la dependencia como síntoma del 'fracaso', insistiendo en los efectos ambivalentes de las políticas sociales; 4) subrayar la tendencia a la feminización y etnización de la dependencia; y 5) analizar la propensión a asociar precariedad laboral y dependencia. Este artículo intenta dar cuenta del carácter múltiple y cambiante de la dependencia, que no se reduce ni a las personas discapacitadas, ni las mayores, ni al periodo actual.

\section{PALABRAS CLAVE:}

dependencia, representación social, feminización, etnización, care. 


\section{Introducción}

¿Qué es la dependencia? ¿Cómo incluir completamente a las personas dependientes en la comunidad social y política? ¿En qué medida somos dependientes de la atención que nos prestan los demás? ¿Cómo dar cuenta de este aspecto fundamental de cualquier vida humana? La respuesta a estas cuestiones no es nada evidente en unas sociedades en la cuales la autonomía individual y la igualdad entre personas constituyen las normas morales y políticas dominantes. Han sido objeto de numerosas reflexiones en la sociología y en el pensamiento posmoderno contemporáneo, principalmente en los países anglosajones. Situadas en el punto de partida de las éticas del care, han permitido visibilizar unos ámbitos de la experiencia moral y social considerados como marginales por las teorías tradicionales. Han conducido a unas notables reformulaciones de las teorías contemporáneas de la justicia, sobre la base de una antropología política que intenta dar cuenta de la constitución relacional de los actores sociales e intenta responder a su vulnerabilidad. Este artículo se centra básicamente en las representaciones sociales de la dependencia que han generado un debate público y académico sobre el cual se fundamentan esas teorías.

\section{Pensar la dependencia}

La dependencia es una noción compleja y multiforme, susceptible de ser concebida en distintos planos. Evoca, a la vez, la precariedad de la vida corporal y biológica, que se manifiesta durante la primera infancia, la vejez y la enfermedad; el carácter fundamental de las necesidades que es preciso satisfacer para que la vida se mantenga; la fragilidad de las identidades que se constituyen a través las relaciones creadas entre los individuos; pero también la influencia y el poder que tiene sobre las personas el entorno natural, social y relacional del que no pueden extraernos jamás y que no pueden controlar del todo. Más allá de las múltiples formas que puede tomar, que van desde la dependencia afectiva y material del niño hacia sus padres hasta la dependencia social y jurídica del asalariado hacia su empleador, más allá igualmente de sus intensidades variables, que dependen del nivel de necesidad así como de los poderes respectivos de sus miembros, las relaciones de dependencia parecen estar marcadas por una ambivalencia fundamental: entre necesidad y obligación, mutualidad y asimetría.

La definición dada por el diccionario da cuenta de esta oscilación entre una perspectiva que insiste en la 'factualidad' de la dependencia y puede admitir su carácter positivo, y un enfoque que, por el contrario, tiende a hacer equivalentes dependencia y dominación, y que aspira a la emancipación ante cualquier forma de dependencia. Un primer sentido
(Rey, 1992), que aparece al final del siglo XIII, alude a la solidaridad de hecho existente entre dos 0 varios elementos: es dependiente lo que no puede realizarse sin la acción o la intervención de otro elemento. La dependencia hace referencia a una relación necesaria y productiva que une un elemento pasivo y un elemento activo, y a través el cual el primero se realiza. Un segundo sentido, que se desarrolla en el siglo XVI, se refiere, en cambio, a la idea de la influencia ejercida por un individuo sobre otro y al estado de sugestión que resulta de ello: es dependiente el que se encuentra 'bajo la autoridad de alguien'.

Estas definiciones tienen el común el hecho de pensar la dependencia como una relación asimétrica; pero, mientras que, en el primer caso, esta asimetría resulta de una limitación constitutiva, en el segundo, está pensada bajo la perspectiva de la obligación, como un obstáculo que puede y debe ser eliminado. Estas definiciones contienen potencialmente dos modelos de la dependencia que son susceptibles de aclarar, a la vez, la posición de las teorías del care sobre esta cuestión, y su posición singular.

Las teorías del care conciben prioritariamente la dependencia en su dimensión antropológica o existencial, y se inscriben, a ese respecto, en la continuidad de una definición de la dependencia como relación necesaria y potencialmente positiva. Como escribe Fineman (2004): "todos vivimos vidas subvencionadas", lo que significa que ninguna existencia humana puede desplegarse si no está apoyada por unas formas de intervención cuyo vector son las relaciones de dependencia. Esta dependencia fundamental puede ser pensada en referencia a la categoría de necesidad: no somos autosuficientes y dependemos de los demás, de su disponibilidad, de su atención y de su trabajo, para la satisfacción de nuestras necesidades de orden tanto fisiológico (beber, comer, dormir) como emocional (necesidad de cariño, de amor, de reconocimiento). Estas formas de dependencia aparecen muy claramente durante los periodos de nuestra vida en los cuales nuestra capacidad de acción está limitada (infancia, vejez o enfermedad). Pero existen también para las personas consideradas como independientes, que continúan dependiendo de ciertas personas para la satisfacción de sus necesidades emocionales, y siguen estando atrapadas en unas relaciones sociales complejas que mediatizan y permiten satisfacer necesidades fisiológicas fundamentales, así como realizar proyectos de vida que se basan en capacidades complejas.

Otra manera de aclarar esta dimensión fundamental e irreducible de la dependencia consiste en recurrir a la noción de vulnerabilidad: las personas son inicialmente vulnerables y siguen siéndolo. Esta vulnerabilidad debe comprenderse como vulnerabilidad del cuerpo (susceptibilidad a las agresiones físicas, 
a las obligaciones, a las privaciones) y como vulnerabilidad identitaria (susceptibilidad a la indiferencia, al desprecio y a la humillación). Estas formas de vulnerabilidad indican la profundidad de las relaciones que nos vinculan con nuestro entorno relacional y social, la influencia que este entorno ejerce, en el mismo momento en el cual permiten comprender que ciertas relaciones de dependencia pueden constituir una respuesta a la vulnerabilidad y una forma de protección.

\section{La noción de dependencia} funciona como un estigma, $y$, paralelamente, la dependencia sería percibida como la señal de

\section{una anomalía}

La noción de vulnerabilidad, en la medida en que puede ser conceptualizada, a la vez, como el fundamento de la dependencia y como su efecto inducido, permite sin embargo comprender la atracción que ejerce un segundo modelo de la dependencia que la considera, no como el producto de una relación necesaria, sino como el resultado de una relación coactiva; no como el corolario de una condición marcada por la necesidad y la vulnerabilidad, sino como el vector de una vulnerabilidad creciente. En este modelo político o jurídico de la dependencia, ésta es prioritariamente comprendida como una forma de sometimiento que limita la capacidad de acción.

Si el paradigma de la primera concepción era la dependencia del niño, el paradigma de esta segunda sería la dependencia del esclavo. Esta concepción negativa expresa una desconfianza hacia las relaciones de dependencia y se fundamenta en la idea según la cual las relaciones de igualdad deben prevalecer en el ámbito moral y político. No obstante, dificulta la concepción de las relaciones asimétricas: las reduce pura y simplemente a unas formas de dominación, o bien, cuando comprende su necesidad a pesar de todo, es para atribuirles un carácter transitorio o de excepción. Idealmente, las relaciones de dependencia están destinadas a ser superadas, porque son percibidas como amenazas que pesan sobre la autonomía individual.

El esbozo de estos dos modelos permite comprender dos orientaciones generales sobre la dependencia que se oponen en varios aspectos. Ciertamente, se puede considerar que estas orientaciones no se sitúan en el mismo plano, de manera que sea posible, en teoría, articularlas. En la perspectiva de semejante articulación, que hace referencia al debate entre teorías del care y de la justicia, el reto sería precisamente elaborar una concepción de la depen- dencia que subraye la irreductibilidad y el carácter positivo de las relaciones de dependencia, además de tomar en consideración los riesgos de sugestión planteados por cualquier relación asimétrica. No en vano, esta articulación requiere que la noción de dependencia sea purgada de las connotaciones peyorativas que le están asociadas, y que indiquen la prevalencia en las representaciones sociales y en las políticas públicas de la segunda concepción a la que acabamos de aludir.

Que semejante concepción prevalga y obstaculice la tesis del carácter fundamental de la dependencia es lo que indican un cierto número de trabajos sociológicos, que comparten un diagnóstico común: en las representaciones sociales dominantes, la dependencia no aparecería como una figura central e irreducible del vínculo social, sino como el atributo de ciertos colectivos. En este contexto, la dependencia sería asociada a una incapacidad o a una deficiencia individual. Por último, esta concepción negativa de la dependencia contribuiría a fortalecer la ideología de la autonomía concebida, de manera problemática, como autosuficiente. Dicho de otra forma, estos autores están de acuerdo sobre el hecho de que la noción de dependencia, tal y como es entendida por la sociedad, funciona como un estigma (Goffman, 1975: 15), un procedimiento de percepción, de etiquetaje y de exclusión, en virtud del cual los individuos considerados como dependientes serían reducidos a sus incapacidades y, paralelamente, la dependencia sería percibida como la señal de una anomalía.

\section{La dependencia como incapacidad}

¿Quiénes son las personas dependientes? Mientras no se ha precisado ni de qué, ni desde qué punto de vista, esta cuestión parece carecer de sentido. No en vano, en Francia, la categoría de 'personas dependientes' ha adquirido existencia jurídica en el marco de la puesta en marcha de la ley que instaura la prestación específica dependencia (PED) en 1997, a la que sucedió la ley que promulga la ayuda personalizada de autonomía (APA) en 2002. Dirigiéndose a las personas mayores de 60 años y caracterizadas por un nivel de dependencia valorada en referencia a un nivel de incapacidad, la ley de 1997 reforzó cierto número de representaciones corrientes de la dependencia, hasta convertir esta última a la vez en la característica exclusiva de las personas mayores que 'envejecen mal' y en el contexto de unas sociedades amenazadas por el envejecimiento de la población.

Es la historia de esta cristalización, que asiste a la constitución progresiva de una concepción médica y discapacitante de la dependencia, la que Bernard Ennuyer, sociólogo y director de una asociación de ayuda a domicilio para personas mayores, describe 
siguiendo un método genealógico inspirado en la perspectiva foucaldiana. Analizando paralelamente el ámbito de las representaciones sociales (a partir del discurso de los medios de comunicación generalistas y especializados), el campo de los discursos oficiales (informes y textos legislativos) y el de los discursos médicos, durante un periodo que va desde los años sesenta hasta hoy día, Ennuyer intenta mostrar "cómo, a partir de una visión sobre todo médica, individual y discapacitante, la noción de dependencia ha conducido lentamente, pero con seguridad, a una nueva categoría, 'las personas mayores dependientes', estigmatizadas como uno de los problemas de la sociedad actual" (Ennuyer, 2004: 1).

En este proceso en el cual dependencia y vejez se han encontrado progresivamente alineadas una sobre otra, la vejez ha sido sometida a un 'nuevo encerramiento', o a una nueva relegación, no tanto entre las paredes de los hospicios, sino detrás de los enunciados y de las representaciones sociales asociadas al término dependencia. De manera recíproca, el concepto de dependencia ha sido troncado para asociarse únicamente a representaciones negativas que conciernen la 'vejez que está mal'. Así, la dependencia, definida por la ley de 1997 como "el estado de la persona que, a pesar de la atención que puede recibir, necesita ser ayudada para la realización de los actos esenciales de la vida, o requiere una vigilancia regular", sería sin embargo comprendida como un efecto de la edad avanzada y la resultante de las deficiencias físicas y mentales a las que daría necesariamente lugar. Para el individuo, significaría una pérdida de autonomía, que convertiría la ayuda de terceras personas en necesaria, y la vigilancia, en legítima. Para el entorno, significaría la necesidad de recurrir al alojamiento o a una ayuda muy importante. Para la sociedad, sería sinónimo de un incremento del gasto público (Ennuyer, 2004). Así, la dependencia, individualizada y naturalizada, habría sido construida progresivamente como un problema específico, el de la vejez con dificultades.

En el ámbito de las políticas públicas, es posible, según Ennuyer, situar el inicio de esta identificación a principios de los años sesenta con la publicación del Informe Laroque (1962). El término 'dependencia' no aparece todavía, pero la vejez ya es presentada como un problema, menos referido a la organización social y más a cierto colectivo: las personas de la cuarta edad. Además, el Informe Laroque realiza una distinción, que el término 'dependencia' fijará y precisará posteriormente, diferenciando, por un lado, a las 'personas mayores válidas', que corresponden a los individuos de entre 65 y 75 años que serán agrupados al inicio de los años ochenta en la categoría de 'jubilados', todavía activos o que dependen de una política social con finalidad integradora; y, por otro lado, a las 'personas mayores inválidas', ancianos de más de 75 años, que se convertirán en las 'personas mayores dependientes', protegidas por una política médico-social.

La categoría de 'personas mayores dependientes' aparece por primera vez en los textos oficiales en el Informe Arrecks, de 1979, donde se define de la siguiente manera: "cualquier anciano que, víctima de una agresión a su integridad física o psíquica, se encuentra en la imposibilidad de responsabilizarse plenamente de su vida y, por lo tanto, debe recurrir a una tercera persona para realizar los actos ordinarios de la vida". El informe precisa que "conviene subrayar que, de manera general, ese deterioro se incrementa principalmente con la edad, por falta de una prevención precoz y de una atención apropiada". La edad y las variables médicas son elevadas al rango de factores explicativos de la dependencia, concebida como incapacidad, en detrimento de la toma en consideración de los factores sociales en el advenimiento de las incapacidades y la vivencia de la vejez, mientras que otros estudios insisten en la preponderancia de estos factores sociales en el aspecto que toma la vida de las personas después de los 60 años (Leclerc et al., 2000).

Esto permite a Ennuyer datar la puesta en marcha de un paradigma biomédico de la dependencia, caracterizado, desde la perspectiva de las representaciones, por "la imposición de un modelo médico del avance de la edad, asimilando la vejez a un estado necesariamente patológico"; desde la perspectiva de los actores sociales, por la atención a las personas mayores como una categoría específica de actores; $y$ desde la perspectiva política, por el paso de una concepción de la vejez como objetivo social integrador a "una visión médica asistencialista" (Ennuyer, 2004: 95). Mientras que la definición de la dependencia generó cierto debate en el ámbito de las ciencias sociales, asociado a las reflexiones dedicadas a la discapacidad y a su medida, la definición será zanjada adoptando esta concepción 'discapacitadora' y médica, a través la cual la dependencia es definida como un atributo propio de la vejez.

Desde ese punto de vista, la ley de la prestación específica de autonomía de 1997 se encuentra en la continuidad del Informe Arrecks, continuidad de la que da cuenta el número de informes y proyectos de ley que se sucedieron entre 1979 y 1997 sin modificar sustancialmente la definición de la dependencia (Ennuyer, 2004: 184-193). La noción de 'pérdida de autonomía' es progresivamente utilizada para aclarar su sentido. Esta confusión de los registros funcional y moral, que tiende a negar a las personas mayores consideradas como dependientes la capacidad de continuar formulando unos proyectos de vida bajo el pretexto de que ya no pueden, por ejemplo, desplazarse sin ayuda externa, ilustra perfectamente la extensión del estigma a todas las dimensiones de la persona. 
Paralelamente a la reconstrucción de este concepto de dependencia, Ennuyer distingue lo que constituyen, según él, sus principales factores explicativos. Entre ellos, subraya la convergencia entre los intereses de los médicos geriátricos, por una parte, y de la clase política, por otra, así como la ausencia de debate público sobre la cuestión de la dependencia, su definición y las modalidades de atención. Efectivamente, fueron médicos geriátricos que trabajaban en centros de larga estancia quienes propusieron, a partir del inicio de los años setenta, la definición de dependencia como discapacidad, reduciéndola al efecto de deficiencias físicas y mentales (Delomier, 1973), antes de que esta definición se impusiera progresivamente en el ámbito político a través el Informe Arrecks. Según Ennuyer, uno de los aspectos de este debate fue la intervención, en el ámbito de la vejez, de médicos de salud pública que transpusieron al ámbito de la vejez el enfoque elaborado por Wood en la Clasificación Internacional de las Discapacidades, para pensar las consecuencias sociales de las enfermedades crónicas (Henrard, 1979).

No en vano, allí donde Wood (1975) elaboró una secuencia que permitía pensar los efectos sociales de una patología médica, los médicos franceses redujeron la consideración de la vejez a la de desventaja, ratificando en un mismo gesto la 'patologización' de la vejez y el escaso cuestionamiento de sus efectos negativos en términos de desventaja social. Como subraya Wood, esta confusión, que oculta la dimensión social del objeto que pretende analizar, presenta una ventaja evidente: centrándose en la dependencia reducida a sus aspectos médicos y a sus manifestaciones individuales, se dota de un objeto aparentemente medible. No obstante, se trata de un aspecto importante en la perspectiva de la puesta en marcha de una política pública de la vejez, cuya evaluación del coste financiero requiere que se fijen unos objetivos precisos y que se designe un colectivo destinatario claro. Así, los años ochenta y noventa, ven pasar el interés de medir la dependencia a definir el fenómeno, ven aparecer instrumentos de medida (Ennuyer, 2004) que van a permitir una política de atención de las personas mayores, sobre la base de la compensación individual, y, por último, ven reforzar la visión médica de la dependencia.

Reservada a una cierta categoría de población, las personas de la cuarta edad, asociadas a unas representaciones negativas, a la pérdida de autonomía o al problema social, y definidas como un estado marcado por un conjunto de incapacidades comprendidas como unas características individuales, la dependencia tal como se ha impuesto en el ámbito de las políticas de vejez en Francia, funciona así como un estigma. En este contexto, ser catalogado como dependiente supone estar excluido, al menos simbólicamente, de la participación a la vida social, al no poder aspirar a ninguna utilidad social. Desde ese punto de vista, Ennuyer hace una observación interesante: indicando la existencia de dos regímenes paralelos de atención de las discapacidades, la prestación compensación de la discapacidad (PCH), para los menores de 60 años, y desde 2002, la ayuda personalizada de autonomía (APA), para los mayores de 60 años, señala que la expresión 'personas dependientes' es reservada únicamente a estas últimas.

Esta distinción -que no se fundamenta en el tipo de incapacidad padecida, ya que las mismas discapacidades están cubiertas por ambas prestacionesseñala que la edad cronológica juega un papel de variable discriminante en la concepción dominante de la dependencia. Indica también que ambos grupos no están simbólicamente situados de la misma manera frente a las normas sociales vigentes (Ennuyer, 2004: 185-189, 252-255). Las personas 'en situación de discapacidad' se perciben cada vez más como personas capaces, susceptibles de integrarse en la vida social gracias a ajustes institucionales y jurídicos. Ése no es el caso de las personas 'dependientes', definidas por el doble criterio de la edad y la deficiencia, y percibidas como un grupo cuyo crecimiento pone en peligro los equilibrios económicos y sociales presentes. Este punto confirma la carga negativa asociada a la noción de dependencia, la cual grava el debate sobre la creación de un quinto riesgo (la dependencia), asumido por la solidaridad estatal, y que pretende responder a todas las formas de discapacidad, sean cual sean sus causas y la edad de los individuos concernidos (Ennuyer, 2004: 242-248).

\section{La dependencia como síntoma del fracaso}

No en vano, se podría objetar a Ennuyer que la noción de dependencia no está únicamente vincula$\mathrm{da}$, en las representaciones sociales, a las personas mayores. Se utiliza igualmente en discursos más informales para caracterizar la falta de conformidad con las normas sociales dominantes de otros actores sociales; el punto en común entre estas personas y las personas mayores dependientes es que son percibidas como individuos cuya participación en la cooperación social es problemática. Es el caso de los colectivos en situación de precariedad socioeconómica, menores de 60 años, que se benefician de la asistencia social, cuya dependencia económica y social a los servicios de la asistencia puede acabar siendo interiorizada como el atributo constitutivo de una identidad social negativa.

Así lo ponen de manifiesto los trabajos de Paugam (2004), dedicados al itinerario de los beneficiarios de la asistencia social en Francia. Paugam elabora una distinción entre tres colectivos que recurren a los servicios sociales: los vulnerables, los asistidos y los 
marginales. Estos grupos se distinguen por las relaciones que mantienen con los servicios de acción social, que generan unas experiencias propias. Según Paugam, la asistencia regular a los servicios sociales, marcada por la puesta en marcha de un seguimiento de tipo contractual por los trabajadores sociales, la concesión de rentas asociadas a la protección social o a la solidaridad estatal, y la interiorización de roles sociales específicos marcan la entrada en la condición de dependencia. Esta entrada en la dependencia constituye, para los actores sociales que lo experimentan, una prueba humillante que induce una reelaboración de la identidad social y moral. Ésta es la razón por la cual estos últimos elaboran estrategias de distinción social que les permiten sustraerse de la imposición del estigma, intentando diferir la instauración de relaciones de dependencia o minimizar su alcance. Estas estrategias pueden ir de la distinción frente a aquellos cuyo estatus es comparable pero cuyo desacredito es notable, a la participación activa en su estigmatización.

Estas estrategias pueden ser interpretadas como los signos de una necesidad de reconocimiento que tienden a confirmar, por el contrario, el desprecio social asociado a la dependencia socioeconómica. Para los que Paugam denomina los 'asistidos', el reto es no dejarse encerrar en las representaciones peyorativas asociadas a su situación social, las cuales ponen en juego valoraciones negativas que aluden tanto a sus capacidades como a su moralidad. Así, y aunque difiera de la dependencia funcional que pueden presentar las personas mayores desde el punto de vista de sus factores y de sus modalidades, la dependencia socioeconómica hacia la asistencia parece estar marcada negativamente, asociada a las nociones de incapacidad, falta de autonomía, inutilidad social e incluso de parasitismo.

Lo mismo se desprende de los trabajos de un cierto número de teóricos feministas que han analizado la función del término 'dependencia' en el marco más amplio de la crítica del Estado social contemporáneo: su originalidad estriba en el hecho de mostrar en función de qué normas estas figuras negativas de la dependencia han acabado constituyendo las únicas existentes. Constatando, efectivamente, que el término de dependencia jugaba un papel retórico en la crítica del Estado social contemporáneo en Estados Unidos, Nancy Fraser y Linda Gordon (1997) decidieron hacer su genealogía. Desde el punto de vista metodológico, su reflexión se inscribe en la continuidad de la perspectiva foucaldiana, como la de Ennuyer, de la que difiere, sin embargo, en varios aspectos: en primer lugar, el contexto analizado no es el mismo; en segundo lugar, el campo analizado es más amplio, puesto que abarca las políticas sociales en general; en tercer lugar, privilegia el largo plazo, cuando el trabajo de Ennuyer se concentra en un periodo concreto de la historia contemporánea gala (Castel, 1995).
Una misma interrogación (¿quién es identificado como dependiente?) y la puesta de manifiesto de una lógica de estigmatización en la construcción de la dependencia justifican, sin embargo, que aproximemos estos trabajos. Además, los estudios de Fraser y Gordon, y en su prolongación, los de Iris Young permiten interrogar con mayor profundidad la construcción de la norma de autonomía asociada a la devaluación de la dependencia. La reflexión de estas feministas aclara los presupuestos ideológicos de la devaluación de la dependencia, preparando la reevaluación que será realizada en el contexto teórico de las éticas del care. Permite mostrar que, si ciertas formas de dependencia están estigmatizadas, es porque cuestionan una concepción corriente de la dependencia, falsa en la medida en que se fundamenta en una denegación de las relaciones múltiples de dependencia en las cuales los individuos están involucrados.

Fraser y Gordon empiezan recordando que, en la época preindustrial, en el marco de una sociedad jerarquizada donde la pertenencia comunitaria definía el estatus y la identidad de los individuos, no era la dependencia, sino la independencia la que se vía asociada a connotaciones peyorativas: designaba entonces la condición de los individuos sin vínculos comunitarios, tales como los vagabundos. La dependencia, por su parte, es indisolublemente política, económica y legal. Hacía referencia a una forma de sugestión, pero se trataba de la otra cara de la moneda de una forma de integración vinculada a un conjunto de protecciones sociales. Es únicamente en la época industrial cuando la dependencia se convierte en problemática, como consecuencia de las mutaciones ideológicas y económicas cuyos principales rasgos son subrayados por estos autores. Fraser y Gordon se interesan por la manera según la cual se diferencian las dimensiones jurídicas, políticas y económicas de la dependencia, anteriormente confundidas, e insisten en la aparición de un registro inédito de la dependencia: el registro moral o psicológico. Por una parte, la dependencia es redefinida hasta el punto de estar solamente asociada a las relaciones de subordinación de orden no económico; por otra parte, se produce en ese momento la distinción entre formas de dependencia estigmatizadas y formas consideradas naturales.

Fraser y Gordon ponen de relieve la manera según la cual la independencia ha acabado por ser percibida como una condición necesaria de la ciudadanía, que debe permitir el acceso a un salario suficiente.

Mientras que la figura del trabajador asalariado es erigida en norma, los que están excluidos del asalariado son rechazados en la esfera de la dependencia. El pobre vive de la asistencia, el colonizado, el esclavo o el ama de casa que depende de su marido encarnan las diversas figuras de la dependencia y constituyen, como otros tantos contrapuntos, la faceta negativa de la independencia del trabajador 
asalariado. Fraser y Gordon observan el aspecto ilusorio de esta independencia, que se fundamenta en la puesta entre paréntesis de la relación de subordinación que existe entre el empleador y el empleado.

Reconstituyen asimismo la manera según la cual el asalariado ha constituido un punto de referencia ideal para el Estado social en construcción: concentrándose en el periodo del New Deal, subrayan que las políticas sociales han sido definidas en el marco de un sistema de dos vías: la primera abarca los programas que garantizan unas indemnidades de desempleo y unas pensiones de jubilación como justa retribución por el hecho de haber cotizado, mientras que la segunda abarca programas que ofrecen asistencia a los más pobres, repartiendo recursos derivados de los impuestos. Es únicamente en el segundo caso en el que los beneficiarios han sido considerados como 'dependientes' o 'asistidos'. Este sistema dual se ha acompañado de una feminización y una etnización de la dependencia, debido al hecho de que los afroamericanos y las mujeres han sido ampliamente excluidos del primer tipo de programa.

\section{Feminización y etnización de la dependencia}

Esta feminización y etnización se refuerzan en la época posindustrial, y Fraser y Gordon explican por qué se han incrementado con el proceso de individualización: si, en la época industrial, la dependencia ha sido redefinida de manera que excluye las relaciones capitalistas de subordinación, la sociedad posindustrial se caracteriza por un proceso de igualización jurídica y política, de manera que podría parecer haber evacuado todas las dependencias de tipo socioestructural y que, si persisten formas de dependencia, están asociadas a disposiciones individuales. En el registro sociojurídico, la dependencia ha continuado siendo definida como subordinación, pero su alcance ha sido considerablemente modificado.

Mientras que, en la era preindustrial, abarcaba una condición mayoritaria que no estaba infravalorada como tal, ha servido, posteriormente, para designar el estatus de ciertos grupos o ciertas categorías consideradas como desviadas. Como subrayan Fraser y Gordon, la individualización de la dependencia se acompañada de una potenciación de su 'psicologización' y moralización. Todo acontece como si las relaciones sociales de dependencia estuviesen 'absorbidas' en la personalidad, o hipostasiadas como características de grupos o de individuos. Al mismo tiempo, las figuras positivas de la dependencia desaparecen progresivamente, señalando a la vez la imposibilidad de justificar la dependencia en sus dimensiones política y jurídica, y la promoción de un ideario de independencia respecto al cual las relacio- nes de dependencia físicas, sociales y emocionales se convierten en incomprensibles, y las personas dependientes devienen invisibles y desviantes.

La oposición entre independencia y dependencia está asociada a una serie de oposiciones: entre masculino y femenino, público y privado, trabajo asalariado y trabajo doméstico, individuo y comunidad, economía y familia

Fraser y Gordon subrayan la importancia, en este proceso de devaluación de las relaciones de dependencia, de la división sexual del trabajo, que asigna a los hombres la responsabilidad de ganar un salario y a las mujeres, la de asumir la carga del trabajo doméstico. De esta división sexual, encarnada en la organización social por la división entre una esfera pública dedicada al trabajo productivo y una esfera privada dedicada al trabajo reproductivo, se derivan las características personales consideradas como fundamentales: el hombre asalariado interioriza y encarna la independencia asociada a la manera según la cual su función económica está interpretada, mientras que la figura del ama de casa está saturada por la dependencia de aquellos de los que se ocupa. La oposición entre independencia y dependencia está asociada, por lo tanto, a toda una serie de oposiciones que se encuentran en el centro del universo cultural de las sociedades modernas (entre masculino y femenino, público y privado, trabajo asalariado y trabajo doméstico, individuo y comunidad, economía y familia).

Fraser y Gordon ilustran y afinan ese tipo de análisis sobre la feminización de la dependencia, que asigna a las mujeres su misión en el marco de la esfera privada, mostrando cómo, por una parte, la figura de la madre soltera se convirtió, durante la época del New Deal, en la figura esencial de la dependencia respecto al Estado social, y cómo, por otra parte, en la continuidad simultánea de una relativa emancipación femenina y de un proceso de etnización, esa figura ha sido sustituida por la de la 'joven mujer afroamericana'. Esta última figura ocupa ahora en Estados Unidos el espacio simbólico precedentemente ocupado por el ama de casa, el pobre, el colonizado, el esclavo: negra, mujer, pobre, en paro, madre sin estar casada, condensa casi todas las características que se oponen a su independencia. 


\section{Desempleo, precariedad laboral y dependencia}

Iris Young ha prolongado la crítica de la idea de autosuficiencia derivada del modelo del trabajo asalariado propuesto por Fraser y Gordon, y ha sacado sus implicaciones positivas y normativas, desarrollando un análisis de las reformas de ayuda social llevadas a cabo en Estados Unidos durante los años noventa. Estas reformas han firmado el paso del welfare al workfare, sistema en el cual la ayuda social está condicionada a la vuelta al trabajo (Young, 2003). Han obedecido a los siguientes principios, que han sido objeto de un amplio consenso: los receptores de la ayuda social deben trabajar, 'trabajar' significa 'tener un empleo' y el objetivo de la ayuda social es convertir a las personas en autónomas. Señalando la angustia que genera la dependencia, el consenso del que es objeto se fundamenta, según Young, en una concepción de la autonomía como autosuficiencia. El ideario de autosuficiencia, según el cual las relaciones de intercambio son posibles pero no necesarias, seduce mediante la condena de las diferentes formas de sugestión.

Sin embargo, la autosuficiencia sigue siendo inaccesible en el contexto de las sociedades contemporáneas, marcadas por los factores conexos de la complejidad y la interdependencia. Ésta es la razón por la cual se hace referencia al hecho de tener un salario y de no depender de la ayuda social. Pero lo que la retórica de la autosuficiencia oculta es el hecho de que muchos puestos de trabajo procuran un salario que no permite satisfacer sus necesidades $y$ unos empleos, precarios y a tiempo parcial, que favorecen una dependencia del empleado con respecto al empleador. Young pone de manifiesto la función ideológica y disciplinar de esta retorica y de sus dos principios fundamentales: la reducción del trabajo al empleo y la ecuación entre 'tener un empleo' y 'ser autosuficiente'. De ahí que la retórica del condicionamiento de la ayuda social al trabajo dificulte cualquier posibilidad de rechazar un empleo, sean cuales sean sus condiciones, y convierte el acceso a la formación y al aprendizaje en un privilegio. Además, refuerza la infravaloración del trabajo doméstico y la marginación de aquellos que, en virtud de su edad o de una discapacidad, no pueden trabajar tanto tiempo como los demás, ni ser tan móviles.

Si Young critica esta retórica, es porque cierra el universo de los discursos posibles sobre el trabajo, del que es imposible discutir la calidad o el sentido. Contra la reducción de cualquier contribución social al hecho de tener un empleo, Young se refiere al ideario de un trabajo dotado de sentido. Propone conformarse con una definición minimalista de ese ideario, lo que implica que el trabajo que se realiza puede ser reconocido como contribución al bienestar del prójimo: debe poder ser una fuente de orgu- llo y contribuir al desarrollo de las capacidades del trabajador. Pero debe también favorecer su compromiso, ya que el trabajador debe poder participar en la definición de sus objetivos y sus medios de realización. Young alude, por lo tanto, a una norma de autonomía, pero la disocia de cualquier ideario de autosuficiencia.

Efectivamente, la dependencia es percibida negativamente, mientras que las situaciones de dependencia, sean físicas -como en el caso de la discapacidad- o sociales -como en el caso de la asistenciadejan abierta la posibilidad de una autonomía personal comprendida como capacidad para definir los objetivos y proyectos de vida sin padecer la dominación de una tercera persona. Coincidiendo con numerosos trabajos relativos al concepto de autonomía y a la posibilidad de concebirlo en los términos de una autonomía relacional (Stoljar y Mac Kenzie, 2000), Young insiste en el hecho de que semejante autonomía no se opone a la dependencia, en la medida en que tiene como condición el apoyo de los demás y la posibilidad de participar en unos proyectos comunes.

\section{Conclusión}

Estos análisis permiten comprender que la devaluación de la dependencia, que se traduce en figuras sociales estigmatizadas (los beneficiarios de la asistencia, pero también las personas mayores), deriva de la promoción de la figura del trabajador asalariado como figura dominante de la autonomía y de la utilidad social. Esta promoción oculta, evidentemente, el hecho de que la relación salarial constituye asimismo una forma de dependencia y no permite necesariamente alcanzar la autosuficiencia, que constituye la principal dimensión de esta concepción reductora de la autonomía. Así, tanto los beneficiarios de la asistencia como las personas mayores son, al no poder participar en el mercado laboral y en la esfera productiva, redefinidos como una carga. Las consecuencias de esta equivalencia cuestionable no se limitan, sin embargo, a la estigmatización de las personas dependientes. Ésta traduce y refuerza la dificultad de una reflexión colectiva sobre lo que significa la dependencia y en que convendría responder a ella colectivamente.

Se puede dudar de que esta reflexión sea posible mientras predomine la percepción negativa y parcial de la dependencia, que lleva implícita la concepción 'discapacitadora' de la dependencia. No en vano, es precisamente en la medida en que proponen una alternativa a esta concepción de la dependencia, de la que muestran que tiene implicaciones morales, sociales y políticas para el conjunto de los miembros de la comunidad política y no solamente para las personas dependientes, que las teorías del care permiten progresar en semejante dirección. 


\section{Bibliografía}

CASTEL, R. (1995): Les métamorphoses de la question sociale, París, Gallimard.

COLVEZ, A.; y BERNARD, J.-M. (1979): "Enquête épidémiologique sur l'état de santé d'une population de personnes âgées (Haute-Normandie)", Gérontologie et Société, $\mathrm{n}$ 9 9.

DELOMIER, Y. (1973): “Le vieillard dépendant. Approche de la dépendance", Gérontologie, nํㅡ, págs. 19-24.

ENNUYER, B. (2004): Les malentendus de la dépendance. De l'incapacité au lien social, París, Dunod.

FINEMAN, M. (2004): The Autonomy Myth. A Theory of Dependency, Nueva York, New York Press.

FRASER, N.; y GORDON, L. (1997): “A genealogy of dependency. Tracing a key-word of US Welfare State", en FRASER, N.: Justice Interruptus. Critical Reflections on the Post-socialist Condition, Nueva York, Routledge, págs. 121-149.

GOFFMAN, E. (1975): Stigmate. Les usages sociaux du handicap, París, Minuit.

HAUT COMITÉ CONSULTATIF DE LA POPULATION ET DE LA FAMILLE, POLITIQUE DE LA VIEILLESSE (1962): Rapport de la Commission d'Étude des Problèmes de la Vieillesse, présidée par M.P. Laroque, París, La Documentation Française.

HENRARD, J.-C. (1979): “Problèmes conceptuels posés par l'étude de la santé des personnes âgées”, Gérontologie et Société, no 9.
JOUAN, M. (2008): Psychologie morale. Autonomie, responsabilité et rationalité pratique, París, Vrin.

LECLERC, A.; et al. (2000): Les inégalités sociales de santé, París, La Découverte.

PAUGAM, S. (2004): La disqualification sociale. Essai sur la nouvelle pauvreté, París, PUF.

REY, A. (1992): Dictionnaire historique de la langue française, París, Le Robert.

STOLJAR, N.; y MAC KENZIE, C. (2000): Relational Autonomy, Nueva York, Oxford University Press.

URTEAGA, E. (2009): “Fundamentos y organización de las políticas sociales en Francia", Portularia, vol. 9, nㅡㄹ 2, págs. 75-95.

WOOD, P. N. (1975): Classification of Impairments and Han dicaps, Nueva York, Organización Mundial de la Salud.

WOOD, P. N.; y BALDLEY, E. M. (1978): “An epidemiological appraisal of disablement”, en BENET, A. E.: Recent Advances in Community Medicine, Nueva York, Churchill Livingstone, págs. 149-173.

YOUNG, I. M. (2003): "Autonomy, welfare reform and meaningful work", in FEDER, E.; y KITTAY, E. F. (dirs.), The Subject of Care. Feminist Perspectives of Dependecy, Lanham, Rowman \& Littlefield, págs. 40-60. 
